\title{
How to Model the Near-to-the-Carrier Regime and the Lower Knee Frequency of Real RF Oscillators
}

\author{
Arsenia Chorti ${ }^{1,2}$ \\ ${ }^{1}$ Department of Computer Communications, EIS Middlesex University, London, NW4 4BT, UK \\ ${ }^{2}$ EEE Department, University College London, London, WC1E 7JE, UK
}

Correspondence should be addressed to Arsenia Chorti, ersi.chorti@gmail.com

Received 17 August 2009; Accepted 15 October 2009

Academic Editor: Shen-Iuan Liu

Copyright ( 2010 Arsenia Chorti. This is an open access article distributed under the Creative Commons Attribution License, which permits unrestricted use, distribution, and reproduction in any medium, provided the original work is properly cited.

\begin{abstract}
Numerous empirical data demonstrate that real noisy RF oscillators are affected by power-law phase noise. However, until recently, the robust analytic modeling of the deep-into-the-carrier spectral regime of RF oscillators was intangible due to the infinities involved in the relevant power-law regions. In this letter we demonstrate how recent advances in oscillator spectral modeling can be applied to extrapolate the near-to-the-carrier regime as well as estimate the oscillator lower knee frequency of transition between the deep-into-the-carrier regime and the power-law regions of real RF oscillators.
\end{abstract}

\section{Introduction}

RF oscillators play an important role in communication systems as they allow the practical realization of important operations including frequency translation, synchronization, timing, and filtering. Because of their importance, the analysis of noise in real oscillators has been the subject of extensive research efforts [1-5].

It is well established in literature that the oscillator phase noise spectrum follows the power-law [1]:

$$
S(f) \propto \frac{k_{\alpha}}{f^{\alpha}}, \quad \text { with typically } \alpha=0,1,2,3,4 .
$$

Nevertheless, the determination of the deep-into-thecarrier regime through measurements is not straightforward because of the perturbations induced from the electronic equipment involved. On the other hand, the theoretical spectral modeling of $S(f)$ around the oscillation frequency was hindered because of the infinities involved in (1) as $f \rightarrow 0$.

As a result, until recently, the near-to-the-carrier regime was modeled either by a flat spectrum at an arbitrarily defined lower knee frequency or as a Lorentzian [2]. However, recent theoretical work in [4] based on the theory of frequency fluctuations [6] has led to a novel spectral model for the deep-into-the-carrier regime. These findings are employed in the present letter towards the mapping of macroscopic spectral measurements in real oscillators to the deep-into-the carrier regime and the lower frequency of transition.

\section{Oscillator Spectral Model with Power-Law Phase Noise}

Amongst the most widely used assumptions about phase noise are the small angle approximation and the Wiener phase noise model. Both these assumptions lead inevitably to the following paradoxes.

(1) Denoting by $R_{w w}(\tau)$ and $R_{\phi \phi}(\tau)$ the oscillator and phase noise autocorrelation functions, respectively, the small angle approximation infers that the power of a unit amplitude complex oscillator $w(t)$ with

$$
w(t)=e^{j\left(\omega_{0} t+\phi(t)\right)},
$$

where $\phi(t)$ is zero-mean Gaussian phase noise, is larger than unity [4]:

$$
R_{w w}(0)=1+R_{\phi \phi}(0) .
$$

This paradox depicts that the small angle approximation is not valid for frequencies around the oscillation frequency where power concentration is high. 
(2) The effect of $1 / f$ flicker phase noise in the nearcarrier oscillator Power Spectral Density (PSD) has so far been approximated either by a constant or by a Lorentzian [2] by assuming an arbitrary cutoff frequency below which flicker phase noise has a flat spectral density. Yet, such a cut-off frequency has never been measured.

(3) It is well established that measured oscillator spectra depict the existence of $k_{3} / f^{3}$ regions while $k_{4} / f^{4}$ regions have also been observed [7]. These regions contradict with the Wiener phase noise model while energy concentration in them furthermore challenges the small angle approximation.

The aforementioned ambiguities result from the fact that the deep-into-the-carrier spectral regime of real RF oscillators cannot be measured to arbitrary accuracy because of the intrinsic phase noise in the electronic circuits used in any experimental set-up. Recent work in $[4,5]$ has introduced a comprehensive oscillator spectral model that accounts for the effect in the near-carrier frequency region of the main power-law phase noise processes. In this work, it was demonstrated that phase noise close to the carrier determines the deep-into-the-carrier regime.

The analysis presented in $[4,5]$ indicates that phase noise can be efficiently approximated as the sum of power-law processes resulting from integration of frequency fluctuations. At relatively high frequencies where the small angle approximation is valid, the oscillator PSD coincides with the phase noise PSD. In regard to the deep-into-the carrier regime, examining the various power-law sub-regions the following cases have been identified [4].

(i) $\alpha=0$. A band-limited flat spectrum phase noise component generates a tone on the oscillator frequency added to a band-limited white-like noise region.

(ii) $\alpha=1+v, v \ll 1$. Flicker phase noise of $k_{1} /|f|^{1+\nu}$ PSD, where $v$ is small, results in an oscillator with finite variance. The dominant side-band spectral component follows a $k_{1} /|f|^{1+v}$ characteristic, while the PSD on the carrier frequency is finite and expressed as

$$
S_{w_{1}}(0)=8 \pi \frac{\Gamma(1 / \nu)}{\nu} \sqrt[v]{-\frac{\Gamma((1+\nu) / 2)}{\sqrt{\pi} k_{1} \Gamma(-v / 2)}} .
$$

(iii) $\alpha=3-\nu, \nu \ll 1$ and $\alpha=4$. Long correlated events in the oscillator phase generate Gaussianlike components in the oscillator PSD around the oscillation frequency. $k_{3} /|f|^{3}$ and $k_{4} /|f|^{4}$ phase noise are such processes.

(iv) $\alpha=2$. Short correlated events in the oscillator phase give rise to Lorentzian components in the oscillator PSD. $k_{2} /|f|^{2}$ phase noise is such a process. Most of the available measurements indicate that long correlated events tend to dominate the near-carrier regime, that is, $k_{4}>k_{3}>k_{2}$.

\section{Deep-into-the-Carrier Regime}

Based on the previous remarks, ambiguities about the nearcarrier regime can be solved and an approximate model can be derived. Starting with phase noise resulting from the addition of uncorrelated power-law phase noise processes $\phi_{\alpha}$ with spectra $k_{\alpha} / f^{\alpha}, \alpha \in\{0,1,2,3,4\}$,

$$
\phi(t)=\sum_{\alpha=0}^{4} \phi_{\alpha}(t)
$$

is deduced that

$$
w(t)=e^{j \omega_{0} t} \prod_{\alpha=0}^{4} w_{\alpha}(t),
$$

where $w_{\alpha}(t)=e^{j \phi_{\alpha}(t)}$. Since the processes $\phi_{\alpha}(t), \alpha \in$ $\{0,1,2,3,4\}$ are uncorrelated, the PSD of the oscillator random process $w(t)$ can be expressed as the convolution of the relevant near-carrier subspectra $S_{w_{\alpha}}(f)$ :

$$
S_{w}(f)=S_{w_{0}}(f) \otimes S_{w_{1}}(f) \otimes S_{w_{2}}(f) \otimes S_{w_{3}}(f) \otimes S_{w_{4}}(f),
$$

where $\otimes$ denotes convolution. At frequencies where the small angle approximation is applicable we can on the other hand express the PSD as the sum of the power-law subspectra $k_{\alpha} / f^{\alpha}$.

As a result of the preceding analysis, the near-carrier PSD is expressed as the convolution of a Dirac delta $(\alpha=0)$, a very high and narrow region $(\alpha=1)$, a narrow Lorentzian region $(\alpha=2)$, and two Gaussian $(\alpha=3,4)$ regions. The convolution of the two Gaussians with the Dirac delta and the flicker noise PSD generates a Gaussian region. Further convolution with the Lorentzian produces the overall PSD regime in the near-carrier frequency region. In that context, based on the fact that measurements depict that $k_{2} \ll k_{4}$, the resulting Gaussian region is notably wider than the Lorentzian. Consequently, their convolution will result in an approximately Gaussian PSD [4].

The proposed enhanced oscillator model includes

(1) a Gaussian-like region near the carrier frequency, as an approximation of the convolution of the nearcarrier subspectra,

(2) a sequence of power-law regions, in accordance with the small angle approximation.

The model is depicted in Figure 1 and assumes that the oscillator can be treated as a Wide Sense Stationary (WSS) random process (rp), inferring that flicker phase noise sources generate finite variance noise terms. The onesided oscillator spectrum at a frequency offset $f$ from the oscillation frequency can be expressed as [4]:

$$
\begin{aligned}
S_{w}(f)= & \left.\frac{1}{\sqrt{2 \pi} \Omega} e^{-\left(2 \pi^{2} f^{2} / \Omega^{2}\right)}\right|_{0} ^{f_{4}}+\left.\frac{k_{4}}{f^{4}}\right|_{f_{4}} ^{f_{3}}+\left.\frac{k_{3}}{f^{3}}\right|_{f_{3}} ^{f_{2}} \\
& +\left.\frac{k_{2}}{f^{2}}\right|_{f_{2}} ^{f_{1}}+\left.\frac{k_{1}}{f}\right|_{f_{1}} ^{f_{0}}+\left.k_{0}\right|_{f_{0}} ^{\omega_{B}} .
\end{aligned}
$$




\section{Evaluation of the Spectral Parameters from Measurements}

The parameters $k_{\alpha}$ of the respective power-law regions are considered to be available from spectral measurements of the oscillator phase noise. The frequencies of transition between the power-law regions $f_{r}, r=0,1,2,3$ as well as the variance $\Omega^{2}$ of the Gaussian region and the lower knee frequency $f_{4}$ between the power-law and the Gaussian deep-into-the carrier regime can then be evaluated from the following set of equations:

$$
\begin{gathered}
f_{r}=\frac{k_{r+1}}{k_{r}}, \quad r=0,1,2,3, \\
\frac{1}{\sqrt{2 \pi} \Omega} e^{-2 \pi^{2} f_{4}^{2} / \Omega^{2}}=\frac{k_{4}}{f_{4}^{4}}, \\
\int_{-\infty}^{\infty} S_{w}(f) \mathrm{d} f=P_{\mathrm{osc}},
\end{gathered}
$$

where $P_{\text {osc }}$ is the oscillator power.

We illustrate the methodology through a real example. The oscillator PSD measurements are provided in $[8$, Figure 10]. The PSD has a $k_{4} / f^{4}$ region close to the carrier followed by a $k_{3} / f^{3}$ and a $k_{2} / f^{2}$ region. Based on the available data we extrapolate the following values for the parameters $k_{\alpha}$ :

$$
\begin{array}{r}
k_{4}=31.62, \quad k_{3}=0.3162, \quad k_{2}=3.16 \cdot 10^{-5}, \\
\text { while } f_{3}=100 \mathrm{~Hz}, \quad f_{2}=10 \mathrm{kHz} .
\end{array}
$$

For the derivation of $f_{4}$ and $\Omega$, we assume that the oscillator is bandlimited at $f_{B}=1 \mathrm{MHz}$. (Although the cut-off frequency of the channel selection filter is arbitrarily set to $\omega_{B}=1 \mathrm{MHz}$, this choice does not alter the results as the contribution of the respective PSD region in the overall oscillator power is negligible.) Furthermore, since the PSD measurements are provided in $\mathrm{dBc} / \mathrm{Hz}$, we have $P_{\text {osc }}=1$. To evaluate the unknowns $f_{4}$ and $\Omega$ we need to solve the system of (10) and

$$
\begin{gathered}
\frac{1}{4 \pi} \operatorname{erf}\left(\frac{\sqrt{2} \pi f_{4}}{\Omega}\right)+\frac{k_{4}\left(f_{3}^{3}-f_{4}^{3}\right)}{3 f_{3}^{3} f_{4}^{3}}+\frac{k_{3}\left(f_{2}^{2}-f_{3}^{2}\right)}{2 f_{2}^{2} f_{3}^{2}} \\
+\frac{k_{2}\left(\omega_{B}-f_{2}\right)}{\omega_{B} f_{2}}=\frac{1}{2} .
\end{gathered}
$$

Setting $x_{0}=\left(\sqrt{2} \pi f_{4}\right) / \Omega$ and substituting in (10) yields

$$
f_{4}^{3}=\frac{2 \pi \sqrt{\pi} k_{4} e^{x_{0}^{2}}}{x_{0}} .
$$

By substitution of (14) into (13) we obtain an equation with the single unknown $x_{0}$ that can be solved numerically. It is then straightforward to evaluate $f_{4}$ and $\Omega$. This approach yields the following results:

$$
\begin{aligned}
& f_{4}=13.59 \mathrm{~Hz}, \\
& \Omega=421.58 \text { or } \Omega=38.96 .
\end{aligned}
$$

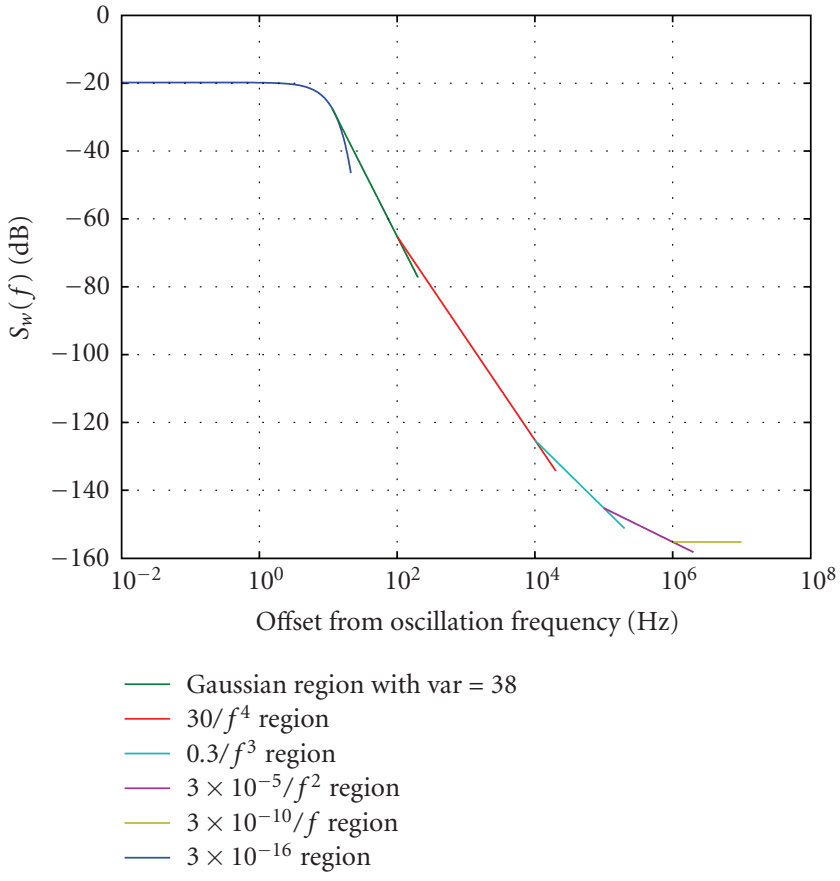

FIGURE 1: Enhanced oscillator spectral model for $k_{4}=30, k_{3}=$ $0.3, k_{2}=3 \cdot 10^{-5}, k_{1}=3 \cdot 10^{-10}$, and $k_{0}=3 \cdot 10^{-16}$. The near-tothe-carrier regime is Gaussian with $\Omega=38$, followed by a sequence of power-law regions with frequencies of transition $f_{4}=11.3 \mathrm{~Hz}$, and $f_{3}=100 \mathrm{~Hz}, f_{2}=10^{4} \mathrm{~Hz}, f_{1}=10^{5} \mathrm{~Hz}$ while the bandwidth of the system is assumed to be $f_{B}=10^{7} \mathrm{~Hz}$.

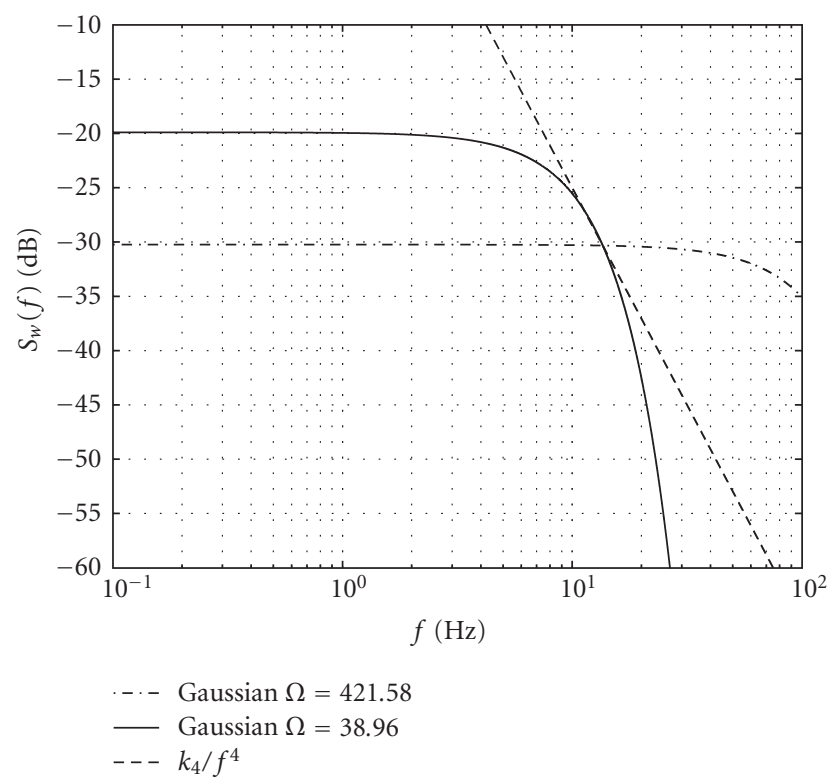

FIGURE 2: Gaussian regions with $\Omega=421.58$ and $\Omega=38.96$ along with the $31.62 / f^{4}$ region.

In Figure 2 we depict the power law region $k_{4} / f^{4}$ along with the two Gaussian regions for $\Omega=421.58$ and $\Omega=38.96$, respectively.

It is clear that the Gaussian with the smallest standard deviation $\Omega=38.96$, provides the best fit to the spectral 
data, so that the one-sided oscillator PSD can be analytically represented as

$$
\begin{aligned}
S_{w}(f)= & \left.\frac{1}{\sqrt{2 \pi} 38.96} e^{-2 \pi^{2} f^{2} / 38.96^{2}}\right|_{0 \mathrm{~Hz}} ^{13.59 \mathrm{~Hz}}+\left.\frac{31.62}{f^{4}}\right|_{13.59 \mathrm{~Hz}} ^{100 \mathrm{~Hz}} \\
& +\left.\frac{0.316}{f^{3}}\right|_{100 \mathrm{~Hz}} ^{10 \mathrm{kHz}}+\left.\frac{3.162 \cdot 10^{-5}}{f^{2}}\right|_{10 \mathrm{kHz}} ^{1 \mathrm{MHz}}
\end{aligned}
$$

\section{Conclusions}

In this letter we demonstrated how macroscopic measurements of an oscillator PSD can provide a robust modeling of the deep-into-the-carrier regime as well as of the lower frequency of transition between the former and the powerlaw regions. This approach overcomes the arbitrariness in oscillator spectral modeling and can be employed when analytic models are required. The range of applicability of the proposed method includes all oscillators that can be treated as WSS rps.

\section{References}

[1] D. B. Leeson, "A simple model of feedback oscillator noise spectrum," Proceeding of the IEEE, vol. 54, no. 2, pp. 329-330, 1966.

[2] A. Demir, A. Mehrotra, and J. Roychowdhury, "Phase noise in oscillators: a unifying theory and numerical methods for characterization," IEEE Transactions on Circuits and Systems, vol. 47, no. 5, pp. 655-674, 2000.

[3] A. Hajimiri and T. H. Lee, "A general theory of phase noise in electrical oscillators," IEEE Journal of Solid-State Circuits, vol. 33, no. 2, pp. 179-194, 1998.

[4] A. Chorti and M. Brookes, "A spectral model for RF oscillators with power-law phase noise," IEEE Transactions on Circuits and Systems I, vol. 53, no. 9, pp. 1989-1999, 2006.

[5] A. Chorti and M. Brookes, "Resolving near-carrier spectral infinities due to $1 / \mathrm{f}$ phase noise in oscillators," in Proceedings of the IEEE International Conference on Acoustics, Speech and Signal Processing (ICASSP '07), vol. 3, pp. 1005-1008, Honolulu, Hawaii, USA, April 2007.

[6] S. Rytov, Principles of Statistical Radiophysics 2: Correlation Theory of Random Processes, Springer, Berlin, Germany, 1988.

[7] E. Rubiola and V. Giordano, "On the 1/f frequency noise in ultra-stable quartz oscillators," IEEE Transactions on Ultrasonics, Ferroelectrics, and Frequency Control, vol. 54, no. 1, pp. 1522, 2007.

[8] U. L. Rohde and A. K. Poddar, "Noise minimization techniques for RF and MW signal sources," Microwave Journal, vol. 50, no. 9, pp. 136-162, 2007. 

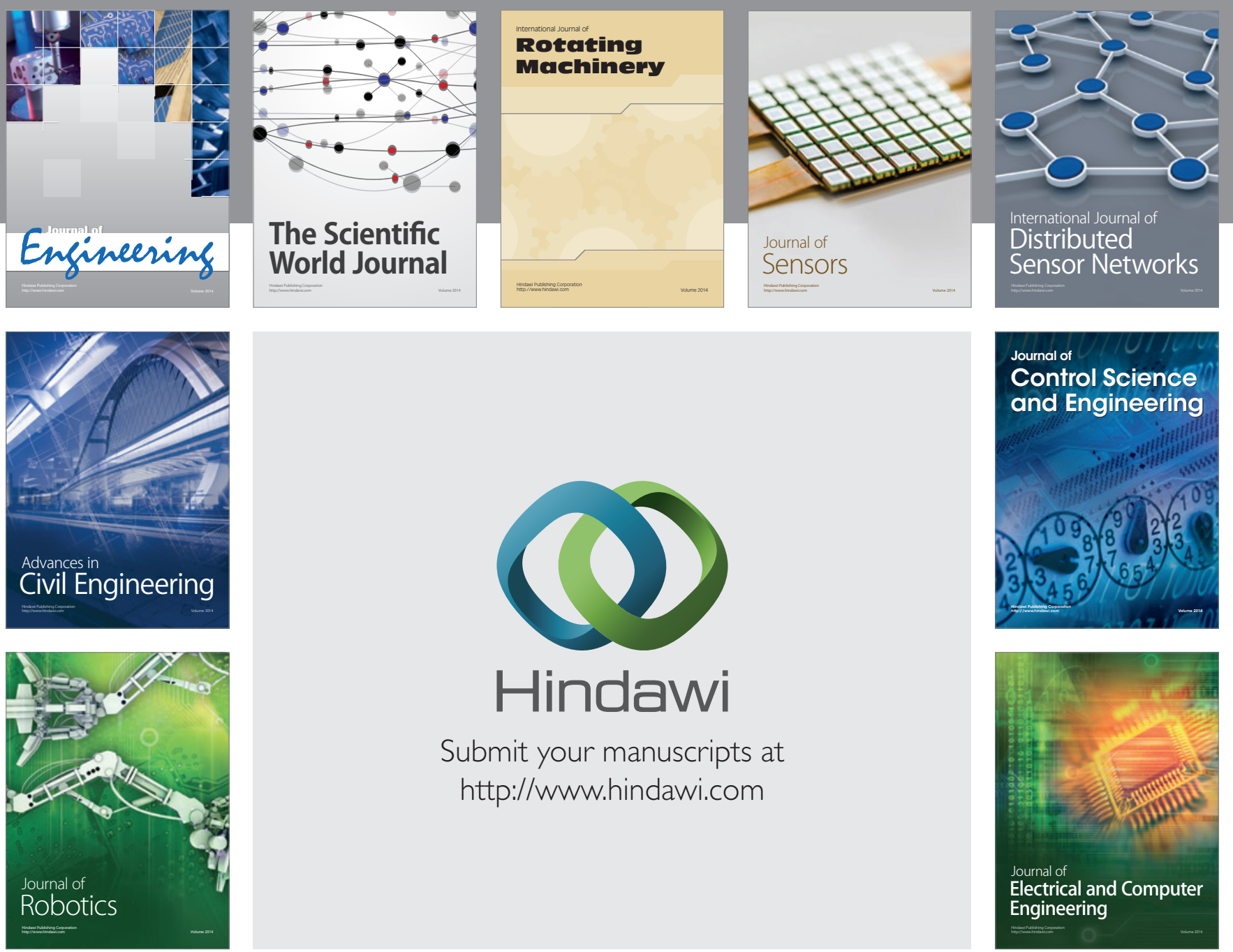

Submit your manuscripts at

http://www.hindawi.com
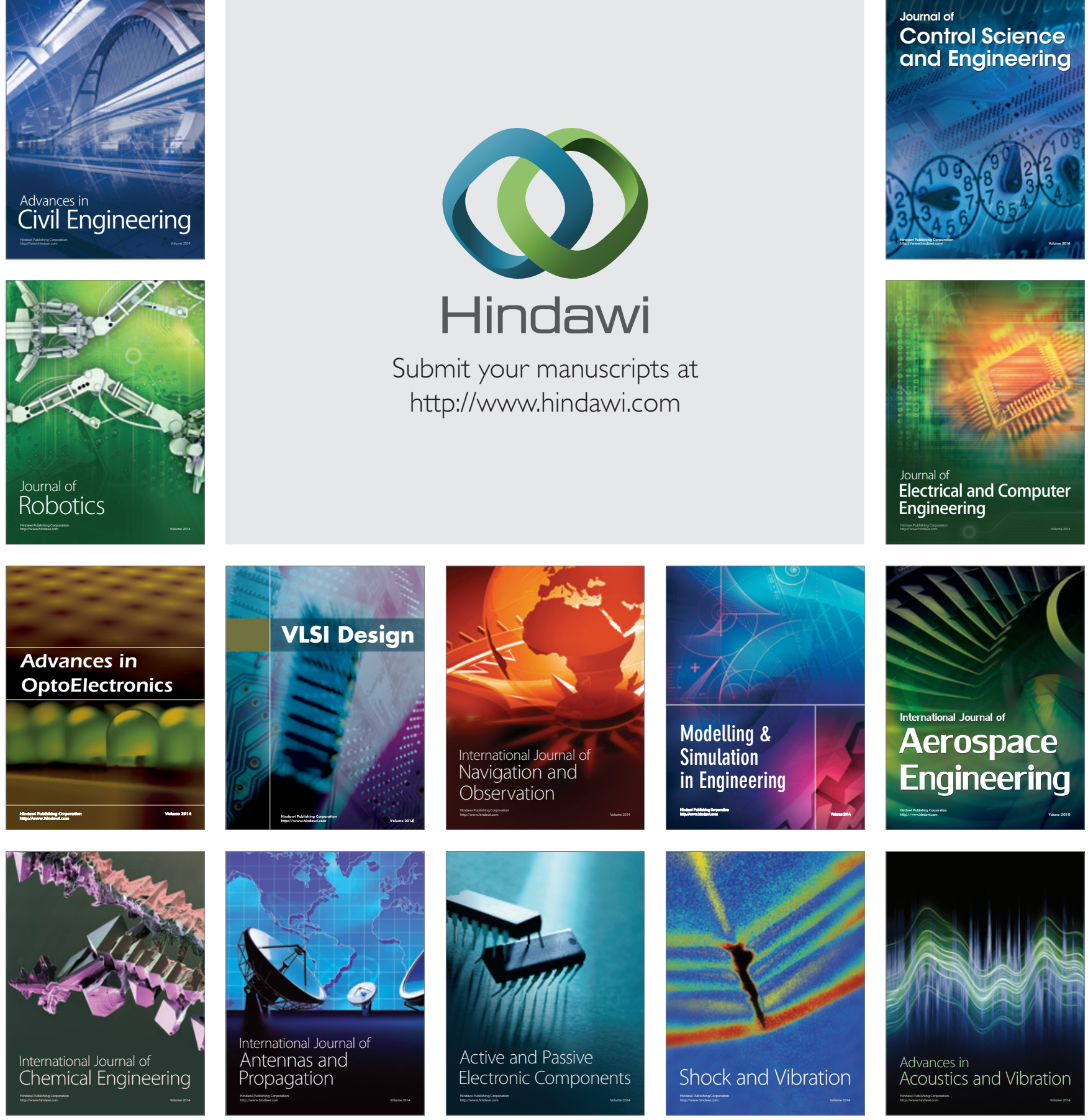